\title{
On some invariant geometric properties in Hele-Shaw flows with small surface tension
}

\author{
PAULA CURT
}

\begin{abstract}
.
In this paper, by applying methods from complex analysis, we analyse the time evolution of the free boundary of a viscous fluid for planar flows in Hele-Shaw cells under injection in the non-zero surface tension case.

We study the invariance in time of $\alpha$-convexity (for $\alpha \in[0,1]$ this is a geometric property which provides a continuous passage from starlikeness to convexity) for bounded domains. In this case we show that the $\alpha$-convexity property of the moving boundary in a Hele-Shaw flow problem with small surface tension is preserved in time for $\alpha \leq 0$.

For unbounded domains (with bounded complement) we prove the invariance in time of convexity.
\end{abstract}

Acknowledgements. This work has been supported by a grant of the Romanian National Authority for Scientific Research, CNCS - UEFISCDI, project number PN-II-ID-PCE-20113-0899.

The author would like to thank the anonymous reviewers for their helpful and constructive comments that greatly contributed to improving the final version of the paper.

\section{REFERENCES}

[1] Curt, P. and Fericean, D., A special class of univalent in Hele-Shaw flow problems, Abstr. Appl. Anal., Vol. 2011, Art. ID 948236, $10 \mathrm{pp}$

[2] Curt, P., Fericean, D. and Groşan, T., $\phi$-like functions in two-dimensions free boundary problems, Mathematica, 63 (2011), No. 76, 121-130

[3] Escher, J. and Simonett, G., On Hele-Shaw models with surface tension, Math. Res. Lett., 3 (1996), 619-642

[4] Escher, J. and Simonett, G., Classical solutions for Hele-Shaw models with surface tension, Adv. Differential Equations, 2 (1997), 467-474

[5] Entov, V. M. and Etingov, P. I., Bubble contraction in Hele-Shaw cells, Quart. J. Mech. Appl. Math., 44 (1991), 507-535

[6] Fericean, D., Strongly $\phi$-like functions of order $\alpha$ in two-dimensions free boundary problems, Applied Math. and Comp., 218 (2012), 7856-7863

[7] Galin, L. A., Unsteady filtration with a free surface, Dokl. Akad. Nauk USSR, 47 (1945), 246-249

[8] Graham, I. and Kohr, G., Geometric Function Theory in One and Higher Dimensions, Marcel Dekker Inc., New York, 2003

[9] Gustafsson, B. and Vasil'ev, A., Conformal and Potential Analysis in Hele-Shaw Cells, Birkhäuser Verlag, 2006

[10] Hohlov, Yu. E., Prokhorov, D. and Vasil'ev, A., On geometrical properties of free boundaries in the Hele-Shaw flows moving boundary problem, Lobachevskii J. Math., 1 (1998), 3-12

[11] Kornev, K. and Vasil'ev, A., Geometric properties of the solutions of a Hele-Shaw type equation, Proc. Amer. Math. Soc., 128 (2000), 2683-2685

[12] Miller, S. S. and Mocanu, P. T., Differential Subordinations:Theory and Applications, Marcel Dekker Inc., New York, 2000

[13] Mocanu, P. T., Une propriété de convexité généralisée dans la théorie de la représentation conforme, Mathematica (Cluj), 11 (1969), No. 34, 127-133

Received: 03.10.2013; In revised form: 12.01.2014; Accepted: 23.04.2014

2010 Mathematics Subject Classification. 30C45, 76D27.

Key words and phrases. Free boundary problem, Hele-Shaw flow, $\alpha$-convex function, starlike function, convex function, surface tension. 
[14] Mocanu, P. T., Bulboacă, T. and Sălăgean, G., Geometric Theory of Univalent Functions, Casa Cărţii de Ştiinţă, Cluj-Napoca, 2006 (in Romanian)

[15] Pandey, J. N., The Hilbert transform of Schwartz distributions and applications, Wiley-Interscience, 1996

[16] Polubarinova-Kochina, P. Ya., On a problem of the motion of the contour of a petroleum shell, Dokl. Akad. Nauk USSR, 47 (1945), 254-257 (in Russian)

[17] Polubarinova-Kochina, P. Ya., Concerning unsteady notions in the theory of filtration, Prikl. Matem. Mech., 9 (1945), 79-90 (in Russian)

[18] Reissig, M., Wolfersdorf, L., A simplified proof for a moving boundary problem for Hele-Shaw flows in the plane, Ark. Mat., 31(1993), 101-116

[19] Vasil'ev, A., Univalent functions in the dynamics of viscous flows, Comp. Methods and Func. Theory, 1 (2001), 311-337

[20] Vasil'ev, A., Univalent functions in two-dimensional free boundary problems, Acta Appl. Math., 79 (2003), $249-280$

[21] Vasil'ev, A. and Markina, I., On the geometry of Hele-Shaw flows with small surface tension, Interfaces and Free Boundaries, 5 (2003), 182-192

[22] Vinogradov, Y. P. and Kufarev, P. P., On a problem of filtration, Akademii Nauk USSR, 12 (1948), 181-198

Department of STATISTICS-ForeCASTS-MATHEMATICS

BABEŞ-BOLYAI UNIVERSITY

CluJ-NAPOCA, ROMANIA

E-mail address: paula.curt@econ.ubbcluj.ro 